\title{
Strong localized variations of the low-altitude energetic electron fluxes in the evening sector near the plasmapause
}

\author{
E. E. Titova ${ }^{1}$, T. A. Yahnina ${ }^{1}$, A. G. Yahnin ${ }^{1}$, B. B. Gvozdevsky ${ }^{1}$, A. A. Lyubchich ${ }^{1}$, V. Yu. Trakhtengets ${ }^{2}$, \\ A. G. Demekhov ${ }^{2}$, J. L. Horwitz ${ }^{3}$, F. Lefeuvre ${ }^{4}$, D. Lagoutte ${ }^{4}$, J. Manninen ${ }^{5}$, T. Turunen ${ }^{5}$ \\ ${ }^{1}$ Polar Geophysical Institute, Murmansk Region, 184200, Apatity, Russia \\ ${ }^{2}$ Insitute of Applied Physics, 603600, Nizhny Novgorod, Russia \\ ${ }^{3}$ The University of Alabama, Huntsville, USA \\ ${ }^{4}$ LPCE/CNRS, Orleans, France \\ ${ }^{5}$ Geophysical Observatory, Sodankylä, Finland
}

Received: 6 January 1997 / Revised: 9 June 1997 / Accepted: 4 July 1997

\begin{abstract}
Specific type of energetic electron precipitation accompanied by a sharp increase in trapped energetic electron flux are found in the data obtained from lowaltitude NOAA satellites. These strongly localized variations of the trapped and precipitated energetic electron flux have been observed in the evening sector near the plasmapause during recovery phase of magnetic storms. Statistical characteristics of these structures as well as the results of comparison with proton precipitation are described. We demonstrate the spatial coincidence of localized electron precipitation with cold plasma gradient and whistler wave intensification measured on board the DE-1 and Aureol-3 satellites. A simultaneous localized sharp increase in both trapped and precipitating electron flux could be a result of significant pitch-angle isotropization of drifting electrons due to their interaction via cyclotron instability with the region of sharp increase in background plasma density.
\end{abstract}

Key words. Ionosphere (particle precipitation; waveparticle interaction) Magnetospheric Physics (plasmasphere)

\section{Introduction}

Various types of localized in latitude precipitation have been observed and studied among the different types of energetic electron precipitation related to magnetic storms and substorms. The ground-based data revealed such phenomena as a narrow band of riometer absorption

Correspondence to: E. E. Titova

E-mail: titova@pgi-ksc.murmansk.su

Fax: + 7-81555-30925 during the substorm growth phase, absorption bursts in the evening sector related to the IPDP events, and a narrow band of anomalous absorption in the evening sector at the equatorial boundary of the eastward electrojet (e.g. Ranta et al., 1981; Pikkarainen et al., 1986; Besprozvannaya et al., 1991). Ground-based observations have shown that typically these kinds of precipitation are observed in the evening-night sector, they have a latitudinal scale of $<1-2^{\circ}$, their longitudinal extension can be as large as tens of degrees, and their lifetime is some tens of minutes.

Different types of localized energetic electron precipitation from the inner magnetosphere in the evening sector have also been selected from the data of lowaltitude satellite observations (e.g. Vampola, 1971; Thorne and Andreoli, 1980; Imhof et al., 1986; Nakamura et al., 1995). In particular, Imhof et al. (1986) considered the electron precipitation spikes that were registered in the narrow L-shell range near the plasmapause. Sometimes the precipitation was registered nearly simultaneously on board different satellites spaced tens of degrees in longitude apart. Duration of the relativistic electron precipitation bursts varies from $<1$ s to 10 $30 \mathrm{~s}$ (Imhof et al., 1986, 1991, 1992; Nakamura et al., 1995)

Thorne and Kennel (1971) suggested a mechanism for the relativistic electron precipitation in the evening and night sectors near the plasmapause. The precipitation from the radiation belt was thought to be related to the electromagnetic ion cyclotron waves. Those waves are generated as a result of overlapping of the ring current protons and the plasmasphere that occurs during the main phase of the magnetic storm. According to Thorne and Kennel (1971) the presence of the proton precipitation is expected during the relativistic electron precipitation. But, as shown by Imhof et al. (1986), localized precipitation of electrons from the inner part of the radiation belt correlates with the proton precipitation spikes only in $31 \%$ of the observed events.

Most of the experimental and theoretical papers mentioned were devoted to localized relativistic electron 
precipitation. In this work, we report and discuss a specific phenomenon of the latitudinally localized evening-side precipitation of the main component of the outer radiation belt electrons $(\mathrm{E}>30 \mathrm{keV})$. This phenomenon has been found in the energetic particle measurements data obtained from the low-altitude NOAA satellites. We selected cases of localized precipitation of energetic electrons observed in close relation to sharp enhancement of the trapped energetic electron flux. We studied the morphological characteristics of these particle precipitation structures as well as their relation to magnetic activity. In addition we considered the relationship between the precipitation structures and cold plasma gradients on board other satellites.

Recently, Trakhtengerts et al. (1996) and Pasmanik et al. (1997) attributed the localized precipitation of energetic electrons in the evening sector to the whistler cyclotron interaction in the region of the sharp gradient of the cold plasma density. Such an interaction of the drifting energetic electrons with the density gradient is expected near the "tongue" of the cold plasma formed in the evening/afternoon sector during the recovery phase of magnetic storm. Our analysis of characteristics of the studied precipitation events shows rather good agreement with these predictions.

\section{Observational results}

The NOAA spacecraft are low-altitude $(850 \mathrm{~km})$ satellites with polar near-circular orbits. The satellites are equipped with an MEPED instrument for measuring particles with energy of $>30 \mathrm{keV}$ (Hill et al., 1985). The instrument has two detectors oriented approximately (when the satellite is at $\mathrm{L}>3$ ) one along and another perpendicularly to the magnetic field. Thus, it can measure both trapped (at the satellite's altitude) and precipitating particles. The MEPED instrument measures the energetic electron in three channels $(>30 \mathrm{keV}$, $>100 \mathrm{keV}$, and $>300 \mathrm{keV}$ ) with a time resolution of $2 \mathrm{~s}$.

Looking through the MEPED instrument data we noted a particular phenomenon shown in Fig. 1 both in logarithmic and linear scales. (The linear scale data are presented to emphasize the structure of trapped population.) The phenomenon is a sharp gradient in the trapped energetic electron flux $\left(J_{t r}\right)$ which is observed near the latitude of the maximal trapped population intensity. The gradient of the trapped electron flux is characterized by flux increase of several times when the satellite latitude increases only by $0.1-0.2^{\circ}$. Because of sharpness of the flux variation we named this feature a "cliff". Simultaneously with the cliff the burst-like enhancement in the precipitation flux $\left(J_{p r}\right)$ is seen in the localized latitudinal region.

We present some statistical characteristics of these phenomena. For the statistical study we used the NOAA data obtained from the periods of 01-10 August 1979 (TIROS-N), 11-31 August 1979 (TIROS-N, NOAA-6), 01-10 May 1980 (NOAA-6), 21-30 November 1981
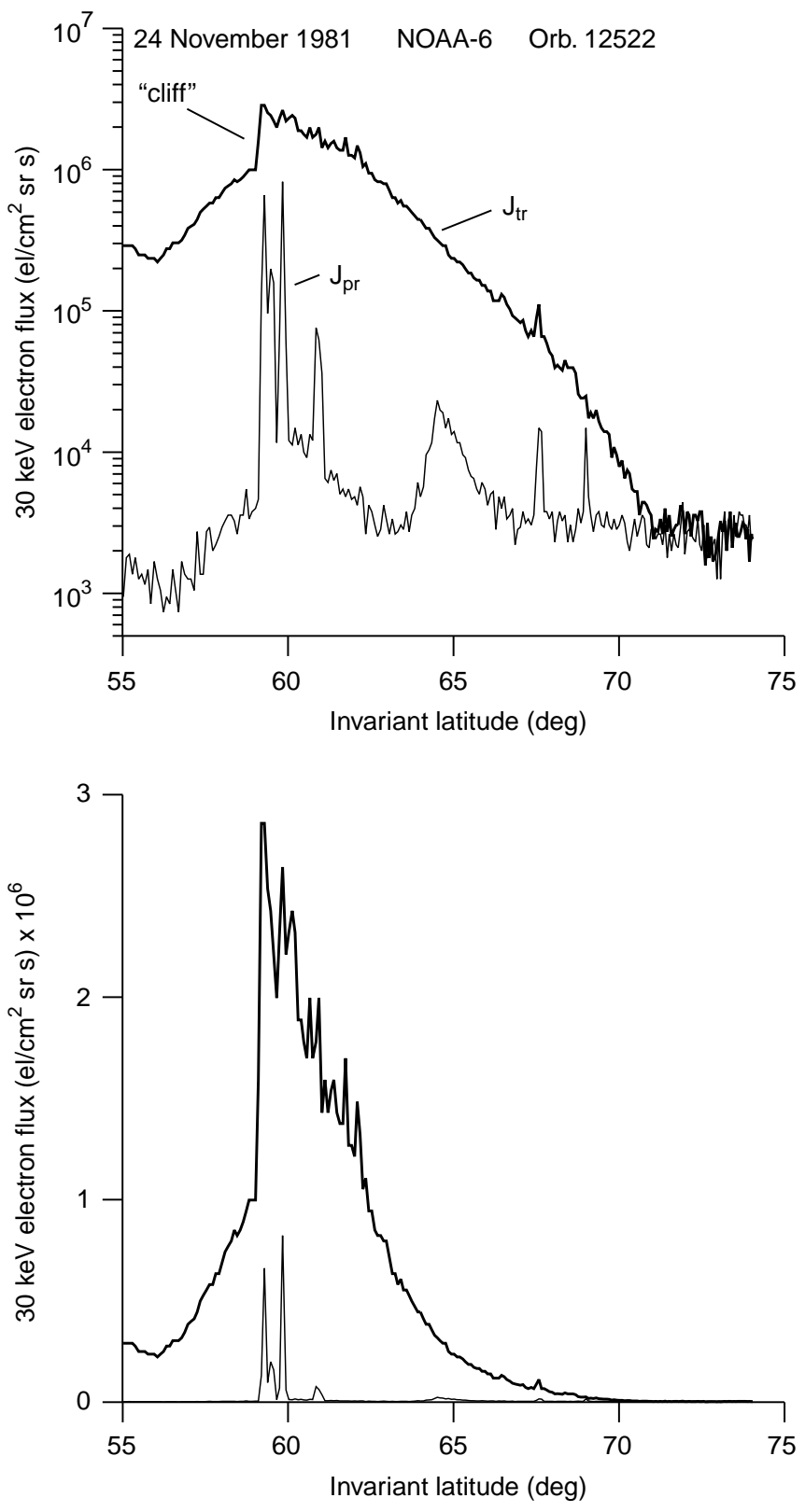

Fig. 1. Example of phenomenon studied in this study. The NOAA satellite data are shown in logarithmic (top) and linear (bottom) scales to emphasize the structure of both the precipitation burst and sharp increase in the trapped flux

(NOAA-6, NOAA-7). For the statistics we selected those cliff events in which the trapped flux increased $>1.5$ times on a latitudinal scale $0.1-0.2^{\circ}$. In all, 114 events have been considered. The data shows that the maximal increase of the flux in the cliff was not more than six fold. The trapped flux gradient in the cliff was always directed poleward. The cliff and localized precipitation were mostly observed at energies $>30 \mathrm{keV}$. Sometimes the phenomenon was simultaneously observed at energies $>100$ and $>300 \mathrm{keV}$, but at these energies it was less pronounced. Thus, the characteristics described in this study were obtained using the data from the detector measuring $>30 \mathrm{keV}$ electrons. 
One of the main characteristics of particle precipitation is the relationship between the intensities of the precipitating and trapped fluxes $\left(J_{p r} / J_{t r}\right)$ characterizing the anisotropy of the particle pitch-angle distribution. In Fig. 2 this relation is shown. In general, the relation is less than 1 , although the tendency to grow is clearly seen when $J_{t r}$ increases.

Figure $3 \mathrm{a}, \mathrm{b}$ presents the dependence of the cliff event observations on MLT and latitude. The MLT dependence exhibits a clear maximum in the evening sector. In fact 92 events $(80.7 \%)$ were observed at 16-22 MLT. In general the cliff and related precipitation bursts were observed at the invariant latitudes $60 \pm 3^{\circ}$, although some dependence of latitude on MLT is also found. To illustrate this dependence we considered the events of simultaneous observations of the cliff by two longitudinally separated satellites. We found six pairs of satellite passes when the time difference between observations of the phenomenon in different longitudinal sectors was as small as $2-22 \mathrm{~min}$. Table 1 summarizes the data on spatial locations of the cliffs observed during these passes. In Fig. 4 those data are plotted, and MLT and latitude of simultaneous observations of the cliff are marked by the same symbols and connected by lines. For all those events we found that the satellite situated at later MLT observed the cliff at lower latitude. This tendency is confirmed also by Fig. 5 which shows the dependence of the cliff latitude on MLT for time intervals when the Dst-index changed insignificantly. In Fig. 5 we combined the observations made by one satellite during sequence of passes when the MLT changed from pass to pass. The data for relatively short UT intervals have been grouped, and the dependence for every group is fitted by straight lines.

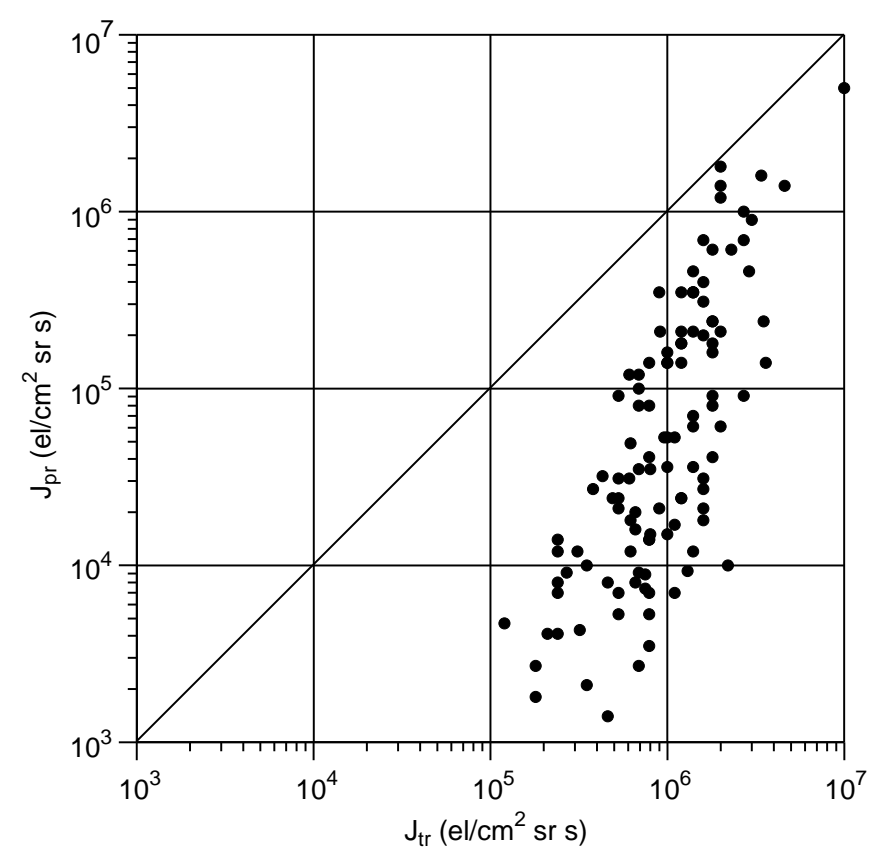

Fig. 2. Relationship between the trapped and precipitating fluxes for the cliff-like events
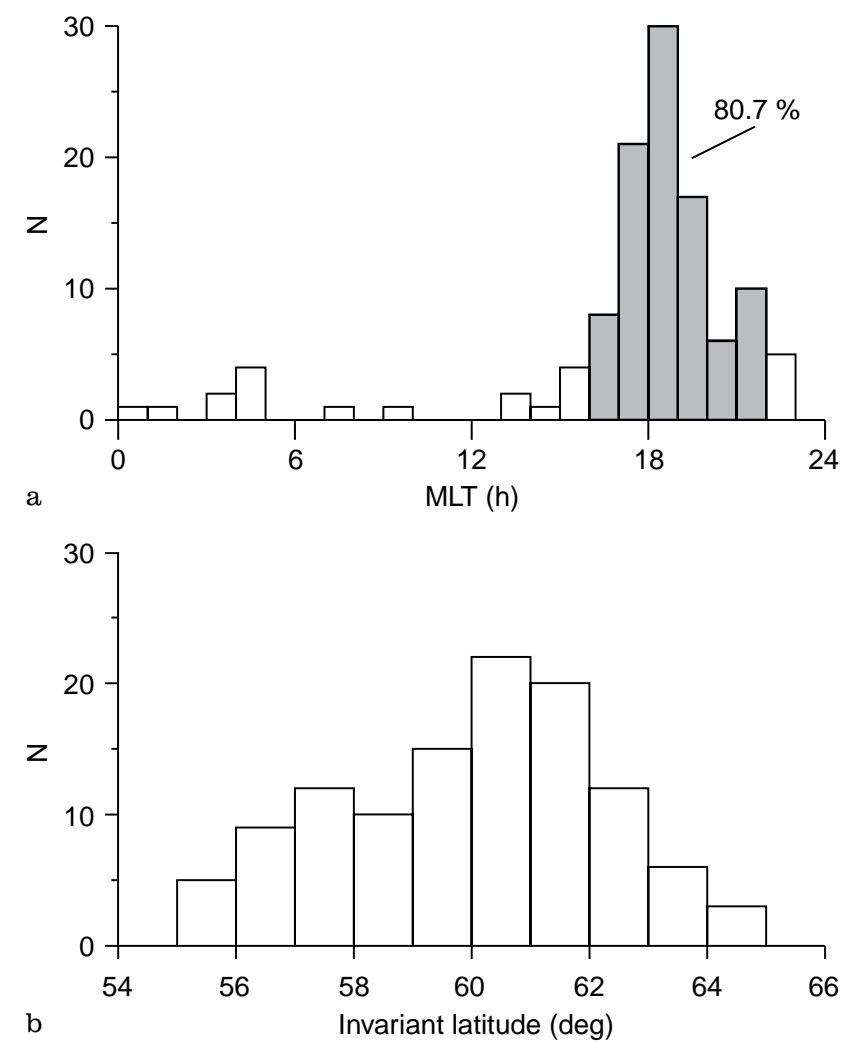

Fig. 3a, b. Occurrence of the cliff phenomena at a MLT and b invariant latitude

As it has been pointed in the Introduction the question on the relationship between electron and proton precipitation is important for interpretation of the precipitation mechanism. We examined the relationship using simultaneous measurements of electrons and protons by MEPED instrument. It has been found that precipitation of energetic $(>30 \mathrm{keV})$ protons are always latitudinally separated from the cliff region. The cliff is typically equatorward of the low-latitude edge of the proton precipitation zone. This is illustrated by Fig. 6, which also shows how the latitudes of the cliff (circles) and low-latitude edge of proton precipitation (crosses)

Table 1. Summary of data on spatial locations of the cliffs

\begin{tabular}{lllrlll}
\hline \multicolumn{2}{l}{ Data } & Satellite & Orbit & UT & $\begin{array}{l}\text { Invariant } \\
\text { latitude } \\
\text { (deg) }\end{array}$ & $\begin{array}{l}\text { MLT } \\
(\mathrm{h})\end{array}$ \\
\hline 1 & & & & & \\
\hline & $79 / 08 / 13$ & NOAA-6 & 671 & 21.41 & 55.35 & 19.75 \\
2 & $79 / 08 / 13$ & TIROS-N & 4293 & 21.49 & 57.68 & 17.58 \\
& & NOAA-6 & 672 & 23.22 & 55.06 & 20.39 \\
3 & \multirow{2}{*}{$79 / 08 / 14$} & TIROS-N & 4294 & 23.32 & 56.60 & 17.95 \\
& & TIROS-N & 4296 & 03.00 & 57.77 & 17.44 \\
4 & \multirow{2}{*}{$79 / 08 / 21$} & NOAA-6 & 675 & 03.17 & 56.28 & 18.05 \\
& & TIROS-N & 4398 & 07.26 & 62.10 & 13.89 \\
5 & \multirow{2}{*}{$81 / 11 / 22$} & NOAA-6 & 777 & 07.35 & 58.63 & 17.77 \\
& & NOAA-7 & 2140 & 02.52 & 60.39 & 16.72 \\
6 & \multirow{2}{*}{$81 / 11 / 24$} & NOAA-6 & 12493 & 02.54 & 58.80 & 17.98 \\
& & NOAA-6 & 12521 & 02.06 & 59.27 & 18.18 \\
& NOAA-7 & 2168 & 02.28 & 59.48 & 16.73 \\
\hline
\end{tabular}






Fig. 4. Dependence of the cliff latitude on MLT for almost simultaneous observations by two longitudinally separated satellites for the events listed in Table 1

depend on Dst (solid and dashed lines, respectively). The data from 16-20 MLT sector were used in Fig. 6.

The relationship of a geophysical phenomenon to the magnetic activity is one of the important characteristics for understanding its nature. In the case of the cliff, 93 events $(81.6 \%)$ were observed during magnetic storms. The rest fell into the intervals after small magnetic disturbances. More detailed consideration of the relationship between the observations of the cliff and magnetic disturbances showed that, in fact, the events were observed during the storm recovery phase. To illustrate this fact the Dst index is plotted for two prolonged intervals covering four magnetic storms in Fig. 7. The black squares mark the times when the cliff events were detected.

\section{Comparison with the other satellite data}

In this section we present the result of comparison of the cliff observations with the data obtained on board the DE-1 and Aureol-3 satellites.

Firstly, we compared the NOAA satellite energetic electron measurements with a set of the DE-1 satellite plasmaspheric ion density profiles published by Horwitz et al. (1990a, b) as well as with a few unpublished profiles. The profiles were calculated from the data of instrument RIMS measuring the cold $(<50 \mathrm{eV})$ ions on board the DE-1 satellite. For the most of events in this set the preceding geomagnetic activity was rather weak, and no cliffs were observed. In agreement with our previous finding, the cliff was observed during the magnetic storm of November 7, 1981. Figure 8a (top) adapted from Fig. 6 of the paper by Horwitz et al. (1990b) presents latitudinal profiles of the ion density and temperature measured at 2026-2053 UT in the 19.720.4 MLT sector. These profiles can be considered as a "multiple plateau" or "multiple plateau with trough" according to the classification by Horwitz et al. (1990a). At the bottom of Fig. 8a the latitudinal profile of energetic electrons is shown. The measurements were taken at about 2000 UT and 19.33 MLT (close to the DE-1 measurements). The latitude of the cliff coincides with the bend of $\mathrm{H}^{+}$density inside the outer boundary of the cold plasma, but poleward of sharp inner gradient.

Another comparison with the DE-1 data was made for the November 24, 1981 event (Fig. 8b). Unfortunately, for this case the analytical program could not be used to obtain precise densities from the RIMS data, even though one can identify density gradients from the count rate fall off in the original DE-1/RIMS data format. (Accurate density values could be obtained only between $50.7-58.3^{\circ}$, where the $\mathrm{H}^{+}$density and temperature changed between $10^{2}-10^{3} \mathrm{~cm}^{-3}$ and $10^{3}-10^{4} \mathrm{~K}$, respectively). In Fig. $8 \mathrm{~b}$ the locations of three outer gradients of cold ions is marked by vertical lines. The outermost marked feature (1) defines the location of the "LEIT" (see, e.g., Horwitz et al. 1990a), i.e., the outer edge of the "observable" cold isotropic rammed ions. The next line (2) indicates the closest sharp density gradient to the LEIT location. The innermost marked gradient (3) is the outer edge of the density trough. The DE-1 measurements were taken at 0250-0300 UT and 18.0-18.2 MLT. Two successive NOAA-6 satellite passes detected the cliff at about 0207 UT and 0350 UT. For the first pass the cliff was observed close to the sector of the DE-1 measurements (MLT $=18.13$ ), for the second pass at 17.66 MLT. Figure $8 \mathrm{~b}$ shows a rather good coincidence of latitudes of the cliff, precipitation bursts, and the DE-1 plasmaspheric ion density gradients.

The comparison with the Aureol-3 satellite data was made for the cliff event observed around 08 UT of November 24, 1981 (Fig. 9). The traces of satellites just coincided, but the Aureol-3 satellite passed the cliff region 8 min later than the NOAA satellite. The altitude of the Aureol-3 satellite was about $1500 \mathrm{~km}$. The trapped and precipitating energetic electron fluxes registered on board the NOAA satellite are shown in the bottom panel of the Fig. 9. The cliff and related burst of precipitation were observed around invariant latitude $57^{\circ}$ and MLT $=21.6$. On the middle panel the plasma frequency determined from the Aureol-3 measurements is shown (see Begin et al., 1982 for description of the instrument on board the satellite). The plasma frequency relates to the electron density in the form of $f_{o e}(\mathrm{MHz}) \approx 9 * \mathrm{~N}^{1 / 2}\left(\mathrm{~m}^{-3}\right)$, so the plasma frequency variations reproduce those of the electron density. Remarkable features seen in the electron density behaviour are the minimum of invariant latitude $\approx 56^{\circ}$ and local maximum at invariant latitude $\approx 57.5^{\circ}$. Unfortunately, the Aureol-3 data are not available at invariant latitude $<55.5^{\circ}$, but undoubtedly that equatorward of the density minimum at invariant latitude $=56^{\circ}$ the increase corresponding to the inner plasmasphere must exists. Note, that the density peak at invariant latitude $=57.5^{\circ}$ is not associated with the auroral particle precipitation. According to the 

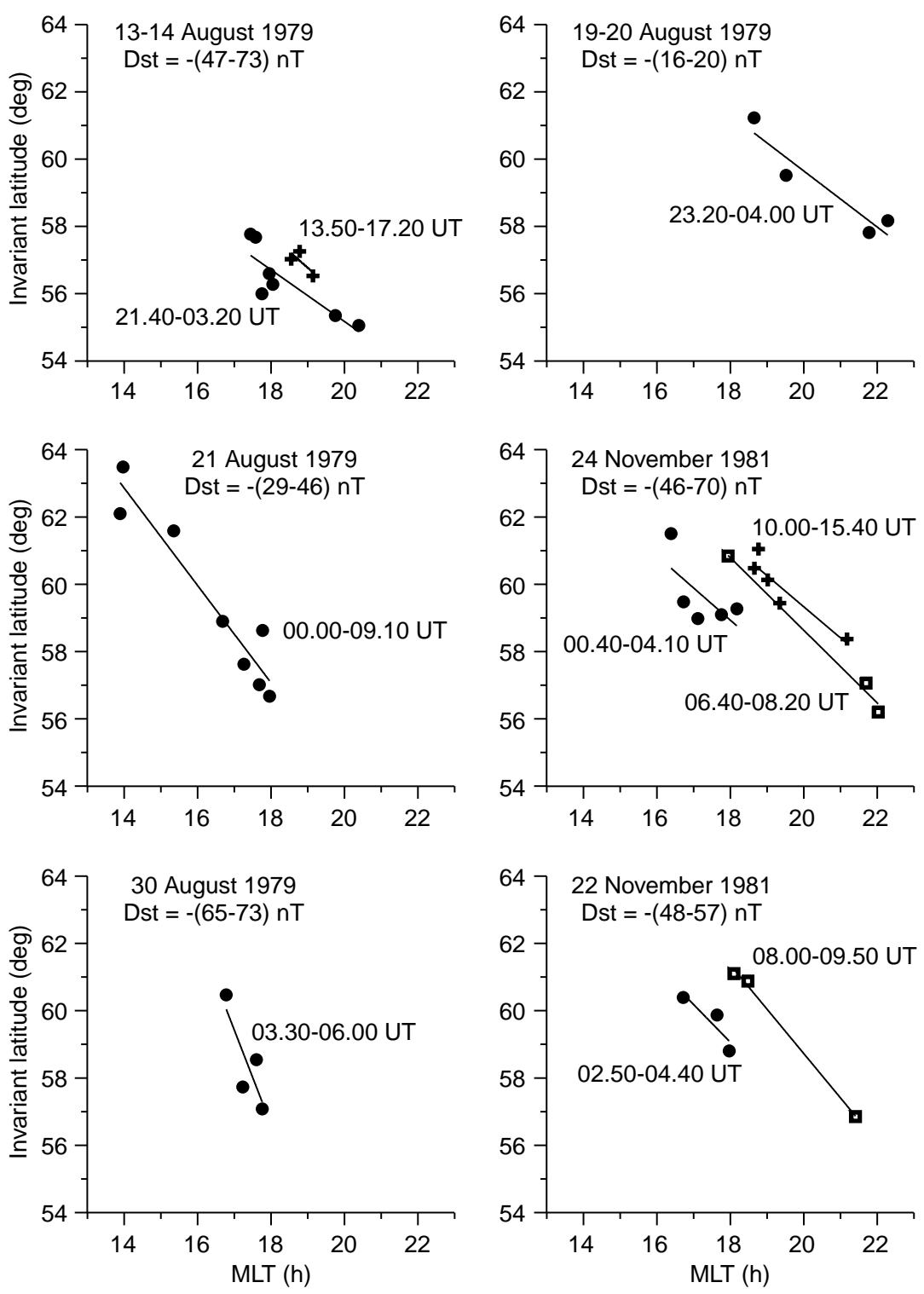

Fig. 5. Dependence of the cliff latitude on MLT for longitudinally spaced satellite passes for prolonged time intervals. Measurements related to different intervals are marked by the different symbols

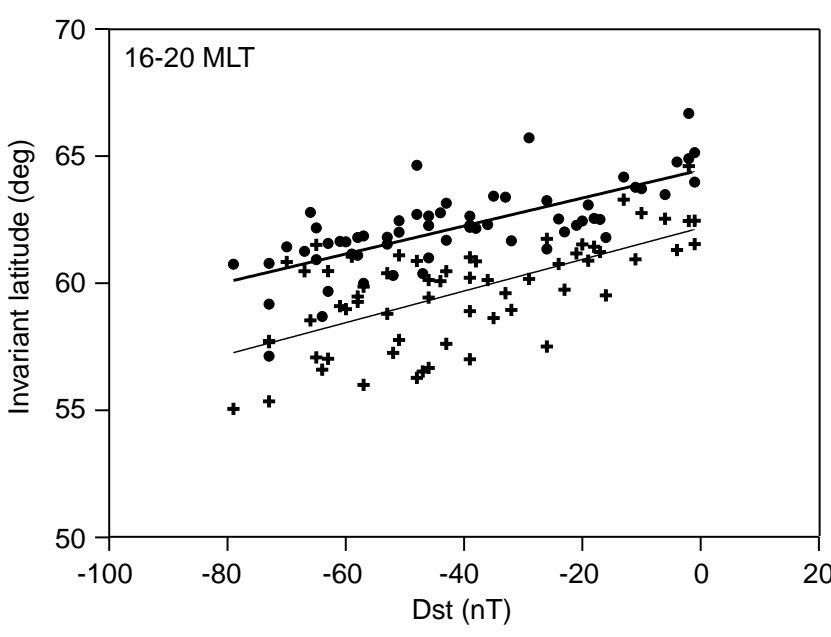

Fig. 6. Latitudes of the cliff (crosses) and low-latitude edge of proton precipitation (dots). Linear fits showing the dependence on Dst are also presented. The data from the 16-20 MLT sector were used low energy $(<30 \mathrm{keV})$ particle measurements on board both NOAA-6 and Aureol-3 (not shown) the precipitation of auroral particles was observed poleward of invariant latitude $=60^{\circ}$ and produced the density enhancement inside the auroral zone, which is also seen in the figure. Thus, the electron density variations in the range of $56-60^{\circ}$ invariant latitude correspond to the fine structure of the outer plasmasphere including the trough and, possibly, detached plasma (e.g. Grebowsky et al., 1974)

From the comparison of the middle and bottom panels of the Fig. 9 it is seen that the cliff latitude coincides with a sharp gradient of the plasma density.

On board the Aureol-3 satellite wave measurements in the frequency range $20-1500 \mathrm{~Hz}$ were carried out (Berthelier et al., 1982). The wave measurements from orbit 840 showed the presence of typical ELF plasmaspheric hiss at frequencies higher than $300 \mathrm{~Hz}$. The top panel of Fig. 9 shows the intensity of the ELF waves registered by the magnetic antenna in frequency band 


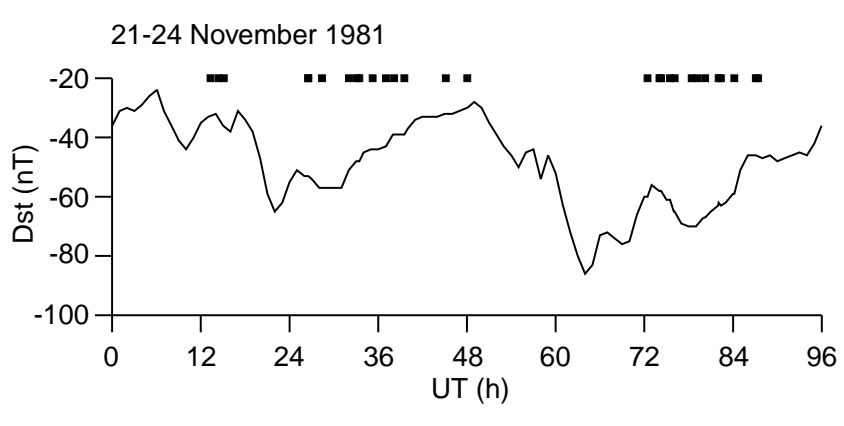

18-21 August 1979

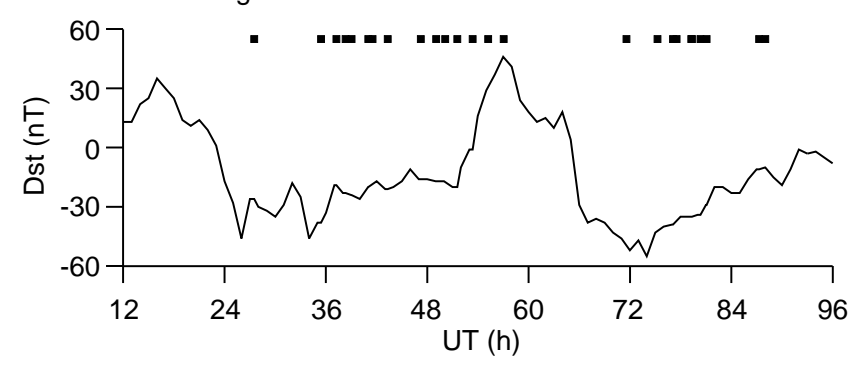

Fig. 7. Relationship between occurrence of the cliff-like phenomena and Dst index. The times of cliff observations are marked by black squares

935-1050 Hz. The black solid area represents the averaged (over period of $0.64 \mathrm{~s}$ ) wave intensity, and the dots show the maximal amplitude. These data show that a strong enhancement of the ELF hiss intensity occurred between invariant latitudes $57^{\circ}$ and $58^{\circ}$ near the latitude of the cliff.

\section{Summary and discussion}

In different studies various types of energetic electron precipitation were considered on the basis of such criteria as: duration of the precipitation bursts, spectral features, relationship with ions, etc. (Vampola, 1971; Thorne and Andreoli, 1980; Imhof et al., 1986, 1991, 1992; Nakamura et al., 1995). In this study we have selected a special class of localized electron precipitation that occurs simultaneously with a sharp gradient (the cliff) in the trapped flux. As far as we know, this cliff-like phenomenon has not been described in previous studies of the energetic electron precipitation from the inner magnetosphere.

We determined position of the cliff because the trapped flux increased by $>1.5$ times over a latitudinal scale $0.1-0.2^{\circ}$. The data confirmed that the greatest increase or surge of the flux in the cliff was not more than six fold.

The cliff has been mainly observed near the maximal trapped flux latitude, and the flux increased polewards. The cliff and localized precipitation were observed at energies $>30 \mathrm{keV}$, but were less pronounced and not so frequent at energies $>100 \mathrm{keV}$ and $300 \mathrm{keV}$.

The main characteristics of these cliff-like precipitations of the energetic electrons which were found in this study are as follows.
1. The relation of intensities in the precipitating and trapped fluxes was less than 1, although the tendency towards the isotropy was noted when the maximal $J_{t r}$ increases (Fig. 2);

2. A total of 92 events $(80.7 \%)$ were observed at $16-22$ MLT (Fig. 3a);

3. The cliff and related precipitation bursts were observed mainly at the invariant latitudes $60 \pm 3^{\circ}$ (Fig. 3b);

4. The latitude of the cliff exhibited the tendency to be smaller for later MLTs (Figs. 4, 5);

5. The cliff events were observed equatorward of the low-latitudinal edge of the proton precipitation (Fig. 6);

6. During magnetic storms 93 events (81.6\%) were observed. The rest are found in the intervals after small magnetic disturbances. More detailed consideration of the relationship between the observations of the cliff and magnetic disturbances shows that, in fact, the events are observed during the storm recovery phase (Fig. 7);

7. A comparison of latitudes of the cliff latitude and the cold plasma gradients measured at different altitudes shows that the cliff corresponds to the cold plasma structures in the outer plasmasphere region (Figs. 8,9)

8. Low-altitude satellite observations show the intensification of the ELF hiss near the cliff.

According to our findings the cliff occurs near the outer plasmasphere. Indeed, in addition to direct comparison with the location of the plasmaspheric cold plasma gradients in Figs. 8 and 9, it can be noted that the average latitude of the cliff is just the same as that of sharp cold plasma gradients obtained from the lowlatitude measurements by the ISEE satellite in the evening sector (Carpenter and Anderson, 1992). Moreover, the MLT-L dependence of the LEIT (outermost cold ion gradient) in the evening sector found by Horwitz et al. (1990a) in the mid-latitude measurements on board DE-1 shows a similar trend to that shown in our Figs. 4 and 5. Thus, we conclude that the studied phenomenon is, likely, related to processes near the cold plasma gradient.

The known mechanism of the energetic electron evening-side precipitation proposed in the paper by Thorne and Kennel (1971) is unable to explain the observed features of the cliff. In fact this mechanism was introduced to explain precipitation of mainly relativistic electrons. As we have noted, in our case $30 \mathrm{keV}$ electrons precipitated more effectively than $100 \mathrm{keV}$ electrons. The mechanism suggested by Thorne and Kennel (1971) should work preferably during the main phase of the magnetic storm when the injected protons propagate into the region occupied by the cold plasmaspheric plasma (see, Fig. 1 of Thorne and Kennel, 1971). In contrast to this prediction our events were observed mainly during the recovery phase of storms. Also we have found that proton and cliff-like electron precipitation are widely spaced in latitude. At the same time the electron and proton precipitation have to coincide in the scheme by Thorne and Kennel (1971). 
24 November 1981 NOAA-6
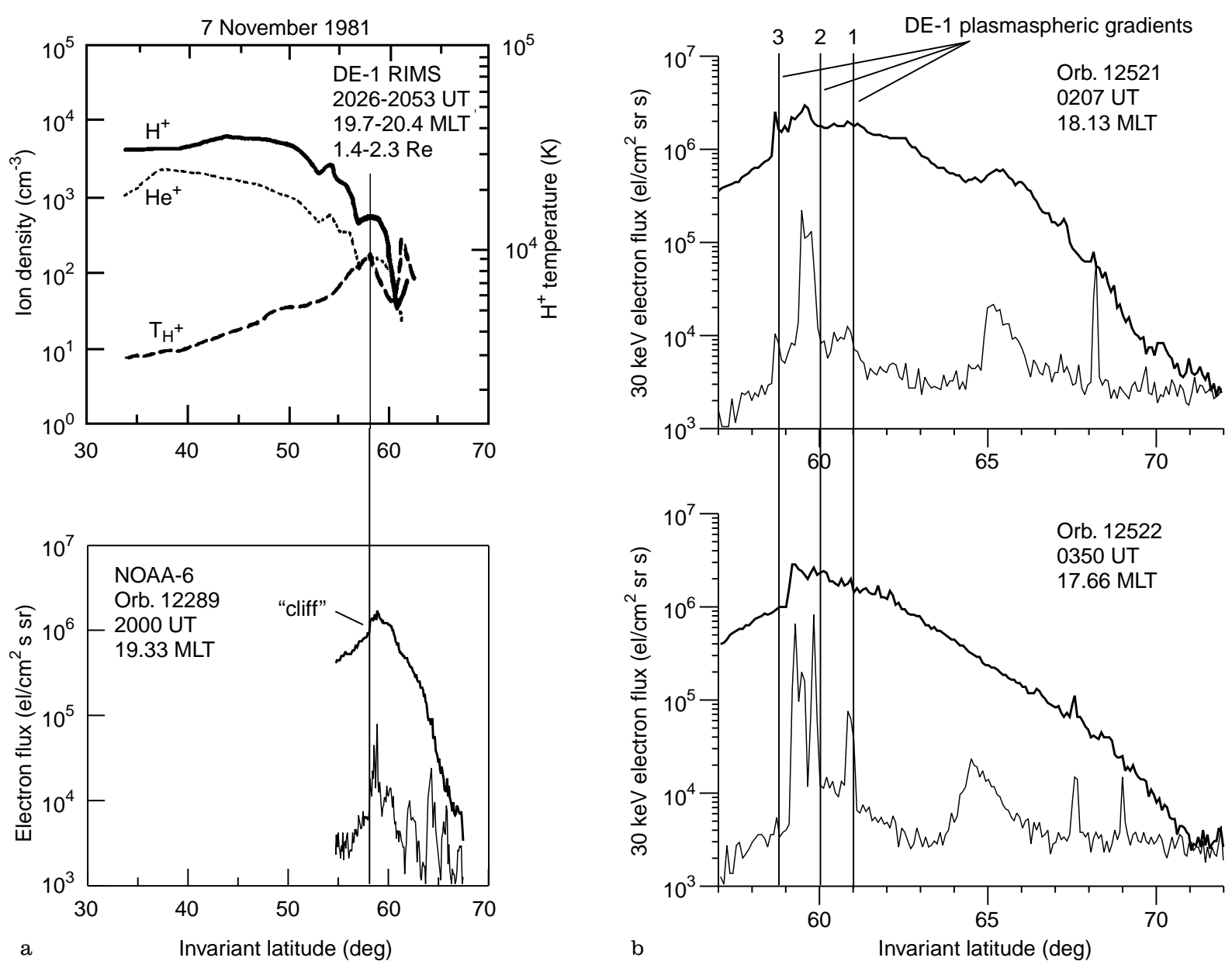

Fig. 8. a Comparison of near simultaneous measurements of the cold plasmaspheric ions on board the DE-1 satellite (top) and the energetic electrons on board the NOAA-6 satellite (bottom); b relationship between the position of the energetic electron precipitation bursts

detected by the NOAA- 6 satellite and gradients of the cold plasma (marked by vertical lines) registered by the RIMS instrument on board the DE-1 satellite

A self-consistent model for the electron precipitation due to the cyclotron interaction of energetic electrons in the region of sharp increase in the cold plasma density has been suggested by Trakhtengerts et al. (1996) and Pasmanik et al. (1997). Interaction of the drifting energetic electrons and cold plasma is expected in the regions where the drift trajectories meet the boundary of the relatively large plasma density. According to this model near the cold plasma density gradient a significant pitch-angle isotropization of energetic electrons occur, and the particle flux in and near the loss cone increases significantly. Precipitating and trapped particles measured on board a low-altitude satellite, in the equatorial plane, have pitch angles lying in and just outside the loss cone, respectively. Thus, at low altitudes, in the region of the cold plasma density gradient one can expect a sharp increase in both precipitating and trapped electron fluxes (or cliff). The mechanism requires the enhanced flux of the drifting energetic electron; this requirement is satisfied during magnetic storms. On the other hand, during magnetic storms the form of the plasmasphere changes dramatically, and the detached cold plasma

structures or "tail-like streamers" appear in the evening and afternoon sectors. Figure 10 presents a schematic view on the cold plasma distribution in the inner magnetosphere during the magnetic storm recovery phase (e.g. Kurita and Hayakawa, 1985; Carpenter et al., 1993) along with drift trajectories of the energetic electrons and protons injected in the midnight sector. The plasmapause itself contracts, and the "tongue" of the cold plasma detaches from the plasmasphere and extends from the night-evening sector to the day side. Like the detached plasma "tongue", the cliff also appears in the evening sector during the recovery phase of magnetic storm. The extension of the "tongue" to the day side allows us to explain the increase in the cliff latitude when MLT decreases.

In Fig. 10 the radial line corresponds to the mapping of a polar orbiting satellite trajectory to the equatorial plane. From the scheme it is clear that the profile of plasma density measured along the trajectory should be similar to that shown in Fig. 8a. Moving from low to high latitudes (from A to B in the scheme) the satellite will sequentially detect the inner plasmapause, "trough" 


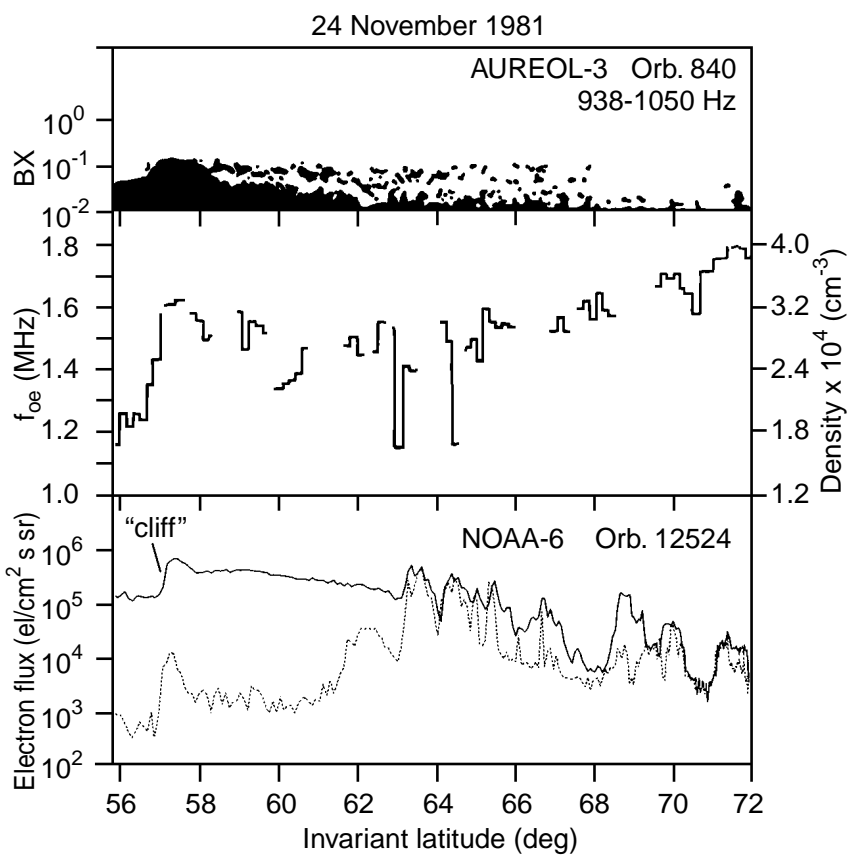

Fig. 9. Comparison of the cliff location obtained from the NOAA satellite measurements (bottom panel) with the location of the ELF wave intensity maximum and sharp gradient of the plasma frequency registered by the Aureol-3 satellite (top and middle panel, respectively)

or "multiple plateau" and the outer edge of the cold plasma structure. According to the scheme, the cliff is expected equatorward of the outermost gradient, close to the outer edge of the density trough.

The fact that the cliff-like phenomenon is regularly observed to be separated from the proton precipitation which are at higher latitudes is also explained in this scheme. Indeed, the westward drifting ions meet the cold plasma on the opposite side of the "tongue". Because the cyclotron interaction of the ions with the Alfven waves depends on the background plasma density the

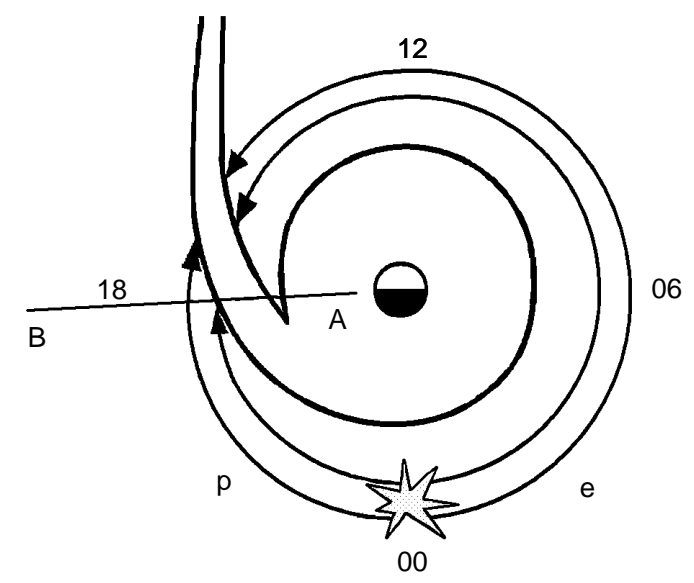

Fig. 10. Schematic presentation showing the interaction of longitudinally drifting energetic particles and the "tongue"-like cold plasma structure formed during storm time on the dusk side of the inner magnetosphere proton precipitation could be expected in this region (Bespalov et al., 1994).

The model by Trakhtengerts et al. (1996) and the one developed by Pasmanik et al. (1997) predicts an enhancement in the intensity of the VLF/ELF waves near the cold plasma gradient in the equatorial magnetosphere. At the same time the existence of the cold plasma gradient is a favourable condition for the propagation of the waves along the magnetic field lines down to low altitudes. Thus, one can expect increased wave intensity near the cold plasma gradient at low altitudes as well. The observations on board the Aureol3 satellite presented in Fig. 9 showed that the latitude of the plasmaspheric ELF hiss intensity maximum was close to that of the plasma density gradient and that of the cliff. As generally accepted, the ELF hiss is a result of the cyclotron wave-particle interaction in the equatorial magnetosphere. Thus, the Aureol-3 data confirm this idea.

The relationship between the cliff and cold plasma gradient allows us to use low-altitude energetic electron observations for remote sensing of the cold plasma in the inner magnetosphere. For example, we can use the result of simultaneous longitudinally spaced observations on two satellites (Table 1 and Fig. 4) for finding the spatial location of the cold plasma "tongue" boundary. Another possibility is to monitor the dynamics of the boundary in time. This can be illustrated by Fig. 5 which shows how the boundary shifts to higher latitudes in the course of the storm recovery phase. Note, that results of calculations made by Kurita and Hayakawa (1985) show similar dynamics of the "tongue" boundary during storm decay.

The experimental data show that the cliff is observed for many hours. Thus, the phenomenon can be used for prolonged monitoring of evolution of the plasmasphere and detached plasma structures.

\section{Conclusion}

The specific type of energetic ( $>30 \mathrm{keV}$ ) electron precipitation related to the sharp gradient of the trapped flux is selected on the basis of data from low-altitude satellites. Characteristics of these precipitation type are investigated, and an agreement has been found between the mechanism of the cyclotron interaction of energetic electrons and the region of sharp enhancement of the cold plasmaspheric plasma density. The increase in the trapped (at the altitude of the satellite) flux is due to isotropization of the pitch angle distribution function near the cold plasma density gradient. Observations of this type of precipitation on board the low-altitude satellites could be used as a diagnostic tool to monitor the location and dynamics of the detached plasmaspheric plasma during magnetic storms.

It is necessary to note that comparison with the cold plasma and wave measurements has been made only for a few particular events. At the same time those measurements are important for identification of the precipitation mechanism. A statistical study of the 
relationship between the cliff and the cold plasma and waves should be undertaken in future.

Acknowledgements. This work was supported in part by INTAS grant 94-2753. Topical Editor K.-H. Glaßmeier thanks R. Nakamura for his help in evaluating this paper.

\section{References}

Begin C., J. F. Karczewski, B. Poirier, R. Debrie, and N. Massevitch, The ARCAD-3 ISOPROBE experiment for high time thermal plasma measurements. Ann. Geophysicae, 38, 615, 1982.

Berthelier J. J., F. Lefeuvre, M. M. Mogilevsky, O. A. Molchanov, Y. I. Galperin, J. F. Karczewski, R. Ney, G. Gogly, C. Guerin, M. Leveque, J.-M. Moreau, and F. X. Sene, Measurements of the VLF electric and magnetic components of waves and DC electric field on board the Aureol-3 spacecraft: the TBF-ONCH experiment, Ann. Geophysicae, 38, 643, 1982.

Bespalov P. A., A. G. Demekhov, A. Grafe, and V. Y. Trakhtengerts, On the role of collective interactions in asymmetric ring current formation, Ann. Geophysicae, 12, 422, 1994.

Besprozvannaya A. S., V. D. Bolotinskaya, A. L. Kotikov, E. I. Nemtsova, O. M. Pirog, O. A. Troshichev, A. V. FrankKamenetsky, and T. I. Shchuka, Structure of auroral zone phenomena from the data of meridional chains of stations: the low ionosphere during the expansion phase of magnetospheric substorms, J. Atmos. Terr. Phys., 53, 275, 1991.

Carpenter D. L., and R. R. Anderson, An ISEE/whistler model of equatorial electron density in the magnetosphere, J. Geophys. Res., 97, 1097, 1992.

Carpenter D. L., B. L. Giles, C. R. Chappell, P. M. E. Decreau, R. R. Anderson, A. M. Persoon, A. J. Smith, Y. Corcuff, and P. Canu, Plasmasphere dynamics in the duskside bulge region: a new look at an old topic, J. Geophys. Res., 98, 19243, 1993.

Grebowsky J. M., Y. Tulunay, and A. J. Chen, Temporal variations in the dawn and dusk midlatitude trough and plasmapause position, Planet. Space. Sci., 22, 1089, 1974.

Hill V. D., D. S. Evans, and H. H. Sauer, TIROS/NOAA satellites space environment monitor, archive tape documentation, NOAA Tech. Mem. ERL SEL-71, 50 pp., Environs. Res. Lab., Boulder, Colo., 1985.

Horwitz J. L., R. H. Comfort, and C. R. Chappell, A statistical characterization of plasmasphere density structure and boundary locations, J. Geophys. Res., 95, 7937, 1990a.

Horwitz J. L., R. H. Comfort, P. G. Richards, M. O. Chandler, C. R. Chappell, P. Anderson, W. B. Hanson, and L. H. Brace, Plasmasphere-ionosphere coupling. 2. Ion composition measurements at plasmaspheric and ionospheric altitudes and comparison with modeling results, J. Geophys. Res., 95, 7949, $1990 \mathrm{~b}$.

Imhof W. L., H. D. Voss, J. B. Reagan, D. W. Datlowe, E. E. Gaines, J. Mobilia, and D. S. Evans, Relativistic electron and energetic ion precipitation spikes near the plasmapause, $J$. Geophys. Res., 91, 3077, 1986.

Imhof W. L., H. D. Voss, J. Mobilia, D. W. Datlowe, and E. E. Gaines, The precipitation of relativistic electrons near the trapping boundary, J. Geophys. Res., 96, 5619, 1991.

Imhof W. L., H. D. Voss, J. Mobilia, D. W. Datlowe, E. E. Gaines, J. P. McGlennon, and U. S. Inan, Relativistic electron microbursts, J. Geophys. Res., 97, 13829, 1992.

Kurita K., and M. Hayakawa, Evaluation of the effectiveness of theoretical model calculation in determining the plasmapause structure, J. Geophys. Res., 57, 130, 1985.

Nakamura R., D. N. Baker, J. B. Blake, S. Kanekal, B. Klecker, and D. Hovestadt, Relativistic electron precipitation enhancements near the outer edge of the radiation belt, Geophys. Res. Lett., 22, 1129, 1995.

Pasmanik D. L., V. Y. Trakhtengerts, A. G. Demekhov, A. A. Lubchich, E. E. Titova, T. A. Yahnina, M. J. Rycroft, J. Manninen, and T. Turunen, A quantitative model for cyclotron wave-particle interaction at the plasmapause, Ann. Geophysicae., 1997, in press.

Pikkarainen T., J. Kangas, H. Ranta, A. Ranta, N. Maltseva, V. Troitskaya, and L. Afanasieva, Riometer absorption events in the evening-to-afternoon sector of the auroral and sub-auroral zone and movements of IPDP SOURCE, J. Atmos. Terr. Phys., 48, 585, 1986.

Ranta H., A. Ranta, R. N. Collis, and J. K. Hargreaves, Development of the auroral absorption substorm: studies of pre-onset phase and sharp onset using an extensive riometer network, Planet. Space Sci., 29, 1287, 1981.

Thorne, R. M., and C. F. Kennel, Relativistic electron precipitation during magnetic storm main phase, J. Geophys. Res., 76, 4446, 1971.

Thorne, R. M., and L. J. Andreoli, Mechanisms for intense relativistic precipitation, in exploration of the polar upper atmosphere, Proc. of the NATO Advanced Study Institute held at Lillehammer, Norway, May 5-6, 1980, p. 381, D. Reidel, Hingham, Mass., 1980.

Trakhtengerts, V. Y., A. A. Lyubchich, A. G. Demekhov, E. E. Titova, T. T. Yahnina, M. J. Ricroft, J. Manninen, and T. Turunen, Cyclotron model for quasi-stationary precipitation of energetic electrons at the plasmapause. Proc. of XIX Apatity seminar "Physics of auroral phenomena", p. 73, Apatity, 1996.

Vampola, A. L. Electron pitch angle scattering in the outer zone during magnetically disturbed times, J. Geophys. Res., 76, 4685, 1971. 